\title{
Existence and Uniqueness Results for Caputo Fractional Differential Equations with Integral Boundary Value Conditions
}

\author{
XUE Yimin*, DAI Zhenxiang, LIU Jie and SU Ying \\ School of Mathematics and Physics, Xuzhou University of Technology, Xuzhou 221018, \\ China.
}

Received 7 September 2017; Accepted 14 December 2017

\begin{abstract}
In the paper, we consider the existence and uniqueness results for Caputo fractional differential equations with integral boundary value condition. The sufficient conditions of existence and uniqueness are obtained by applying the contraction mapping principle, Krasnoselskii's fixed point theorem and Leray-Schauder degree theory, which partly improves and extends the associated results of fractional differential equations. Four examples illustrating our main results are included.
\end{abstract}

AMS Subject Classifications: 26A33, 34A08, 34B18

Chinese Library Classifications: O175.8

Key Words: existence and uniqueness; Caputo derivative; fractional differential equation; fixed point theorem; integral boundary value conditions.

\section{Introduction}

Fractional differential equations can be used to describe many phenomena in a number of fields. For examples in physics, polymer rheology, chemistry, electrodynamics of complex medium, regular variation in thermodynamics, control theory, signal and image processing, biophysics, and so forth. There are many papers dealing with the existence and uniqueness results of boundary value problems for nonlinear fractional differential equations [1-5]. Meanwhile, boundary value problems with integral boundary value conditions of nonlinear fractional differential equations have aroused considerable attention. Boundary value problems with integral boundary value conditions have various

*Corresponding author. Email addresses: xueym@xzit.edu.cn (Y. M. Xue), 402898530@qq.com (Z. X. Dai), 1046550060@qq.com (J. Liu), suyingxzit@163.com (Y. Su) 
applications in population dynamics,chemical engineering, etc. For some recent development on the integral boundary conditions, see the texts [6-9] and the references cited therein.

Recently, in [10] Bai and Lü used classical fixed point theorems to prove the multiple positive solutions for the following nonlinear fractional differential equation

$$
\left\{\begin{array}{l}
D^{\alpha} u(t)+f(t, u(t))=0, \quad 0<t<1 \\
u(0)=u(1)=0
\end{array}\right.
$$

where $1<\alpha \leq 2, D^{\alpha}$ is the Riemann-Liouville fractional derivative of order $\alpha$ and

$$
f \in C([0,1] \times[0, \infty),[0, \infty)) .
$$

In [11], $\mathrm{Xu}$ et al. investigated the existence of positive solutions for the following fractional boundary value problem

$$
\left\{\begin{array}{l}
D^{\alpha} u(t)+f(t, u(t))=0, \quad 0<t<1, \\
u(0)=u^{\prime}(0)=u(1)=u^{\prime}(1)=0,
\end{array}\right.
$$

where $2<\alpha \leq 3, D^{\alpha}$ is a fractional derivative in the sense of Riemann-Liouville and $f \in$ $C([0,1] \times[0, \infty),[0, \infty))$. The existence, multiplicity, uniqueness of positive solutions are established by using some fixed point theorems.

In [12], Cabada and Wang used Guo-Krasnoselskii fixed point theorem to show the existence of positive solutions for a class of nonlinear boundary value problem with integral boundary conditions as

$$
\left\{\begin{array}{l}
{ }^{c} D^{\alpha} u(t)+f(t, u(t))=0, \quad 0<t<1, \\
u(0)=u^{\prime \prime}(0)=0, \quad u(1)=\lambda \int_{0}^{1} u(s) \mathrm{d} s,
\end{array}\right.
$$

where $2<\alpha<3,0<\lambda<2,{ }^{c} D^{\alpha}$ is the Caputo fractional derivative of order $\alpha$, and $f \in$ $C([0,1] \times[0, \infty),[0, \infty))$.

Motivated by the results of [10-12], we consider the existence and uniqueness results for the following Caputo fractional differential equations with integral boundary value condition

$$
\begin{cases}{ }^{c} D^{\alpha} u(t)+f(t, u(t))=0, & 0<t<1, n<\alpha<n+1, \\ u(0)=u^{\prime}(0)=\cdots=u^{(n-2)}(0)=u^{(n)}(0)=0, & \\ u(1)=\lambda \int_{0}^{1} u(s) \mathrm{d} s, & 0<\lambda<n,\end{cases}
$$


where $n \geq 2(n \in \mathbb{N}),{ }^{c} D^{\alpha}$ is the standard Caputo fractional derivative of order $\alpha$, and $f \in C([0,1] \times \mathbb{R}, \mathbb{R})$ is a given function. The contraction mapping principle, Krasnoselskii's fixed point theorem, Leray-Schauder degree theory and Schauder fixed point theorem are applied to prove the sufficient conditions of existence and uniqueness. Our main results partly improves and extends the associated results of [12]. Four examples illustrating our main results are included.

\section{Preliminaries}

For the convenience of the reader, we will demonstrate and study some necessary definitions and theorems which can been founded in [13-18].

Definition 2.1. ([13,14]) For a function $g:[0, \infty) \rightarrow \mathbb{R}$, the Caputo fractional derivative of order $\alpha>0$ is given as

$$
{ }^{c} D^{\alpha} g(t)=\frac{1}{\Gamma(n-\alpha)} \int_{0}^{t}(t-s)^{n-\alpha-1} g^{(n)}(s) \mathrm{d} s,
$$

provided the previous integral exists. where $n=[\alpha]+1,[\alpha]$ denotes the integer part of $\alpha$.

Definition 2.2. ([15,16]) For a function $g:(0, \infty) \rightarrow \mathbb{R}$, the Riemann-Liouville fractional integral of order $\alpha>0$ is the integral

$$
I^{\alpha} g(t)=\frac{1}{\Gamma(\alpha)} \int_{0}^{t}(t-s)^{\alpha-1} g(s) \mathrm{d} s,
$$

provided that the integral of the right-hand side of the previous equation exists.

Lemma 2.1. ([15]) Let $\alpha>0$, then the fractional differential equation ${ }^{c} D^{\alpha} u(t)=0$ has a unique solution given as

$$
u(t)=\sum_{j=0}^{[\alpha]} \frac{u^{(j)}(0)}{\Gamma(j+1)} t^{j} .
$$

Lemma 2.2. ([15]) Let $\alpha>0$, then the integral and derivative of the previous have the composite property as

$$
I^{\alpha}\left\{{ }^{c} D^{\alpha} u(t)\right\}=u(t)-\sum_{j=0}^{[\alpha]} \frac{u^{(j)}(0)}{\Gamma(j+1)} t^{j} .
$$

To obtain the solution of the boundary problem (1.1), we establish the following fractional differential equation with integral boundary value condition

$$
\left\{\begin{array}{l}
{ }^{c} D^{\alpha} u(t)+y(t)=0, \quad 0<t<1, \quad n<\alpha<n+1, \\
u(0)=u^{\prime}(0)=\cdots=u^{(n-2)}(0)=u^{(n)}(0)=0, \\
u(1)=\lambda \int_{0}^{1} u(s) \mathrm{d} s, \quad 0<\lambda<n,
\end{array}\right.
$$


from which, we have the following lemma:

Lemma 2.3. For any $y(t) \in C[0,1], n \geq 2(n \in \mathbb{N}), 0<\lambda<n$, then problem (2.1) has a unique solution given by:

$$
u(t)=\int_{0}^{1} G(t, s) y(s) \mathrm{d} s,
$$

where

$$
G(t, s)=\left\{\begin{array}{l}
\frac{n t^{n-1}(1-s)^{\alpha-1}(\alpha-\lambda+\lambda s)-(n-\lambda) \alpha(t-s)^{\alpha-1}}{(n-\lambda) \Gamma(\alpha+1)}, \quad 0 \leq s \leq t \leq 1, \\
\frac{n t^{n-1}(1-s)^{\alpha-1}(\alpha-\lambda+\lambda s)}{(n-\lambda) \Gamma(\alpha+1)}, \quad 0 \leq t \leq s \leq 1 .
\end{array}\right.
$$

Proof. We may apply Lemma 2.2 to reduce problem (2.1) to an equivalent integral equation:

$$
u(t)=-I^{\alpha} y(t)+\sum_{j=0}^{n} \frac{u^{(j)}(0)}{\Gamma(j+1)} t^{j}=-\int_{0}^{t} \frac{(t-s)^{\alpha-1} y(s)}{\Gamma(\alpha)} \mathrm{d} s+\sum_{j=0}^{n} \frac{u^{(j)}(0)}{\Gamma(j+1)} t^{j} .
$$

Since $u(0)=u^{\prime}(0)=\cdots=u^{(n-2)}(0)=u^{(n)}(0)=0$, we deduce that

$$
u(t)=-\int_{0}^{t} \frac{(t-s)^{\alpha-1} y(s)}{\Gamma(\alpha)} \mathrm{d} s+\frac{u^{(n-1)}(0)}{\Gamma(n)} t^{n-1} .
$$

Using the integral boundary condition $u(1)=\lambda \int_{0}^{1} u(s) \mathrm{d} s$, we find that

$$
u^{(n-1)}(0)=\Gamma(n)\left(\int_{0}^{1} \frac{(1-s)^{\alpha-1} y(s)}{\Gamma(\alpha)} \mathrm{d} s+\lambda \int_{0}^{1} u(s) \mathrm{d} s\right) .
$$

In view of (2.3) and (2.4), yields

$$
u(t)=-\int_{0}^{t} \frac{(t-s)^{\alpha-1} y(s)}{\Gamma(\alpha)} \mathrm{d} s+t^{n-1} \int_{0}^{1} \frac{(1-s)^{\alpha-1} y(s)}{\Gamma(\alpha)} \mathrm{d} s+\lambda t^{n-1} \int_{0}^{1} u(s) \mathrm{d} s .
$$

Now we integrate the previous equality from 0 to 1 on both sides on $t$, yields

$$
\begin{aligned}
\int_{0}^{1} u(t) \mathrm{d} t=-\int_{0}^{1} \int_{0}^{t} \frac{(t-s)^{\alpha-1} y(s)}{\Gamma(\alpha)} \mathrm{d} s \mathrm{~d} t+\int_{0}^{1} \int_{0}^{1} \frac{t^{n-1}(1-s)^{\alpha-1} y(s)}{\Gamma(\alpha)} \mathrm{d} s \mathrm{~d} t \\
+\int_{0}^{1} \lambda t^{n-1} \int_{0}^{1} u(s) \mathrm{d} s \mathrm{~d} t \\
=-\int_{0}^{1} \frac{(1-s)^{\alpha-1} y(s)}{\alpha \Gamma(\alpha)} \mathrm{d} s+\frac{1}{n} \int_{0}^{1} \frac{(1-s)^{\alpha-1} y(s)}{\Gamma(\alpha)} \mathrm{d} s+\frac{\lambda}{n} \int_{0}^{1} u(t) \mathrm{d} t .
\end{aligned}
$$


Therefore, we have

$$
\int_{0}^{1} u(t) \mathrm{d} t=-\frac{n}{n-\lambda} \int_{0}^{1} \frac{(1-s)^{\alpha} y(s)}{\Gamma(\alpha+1)} \mathrm{d} s+\frac{1}{n-\lambda} \int_{0}^{1} \frac{(1-s)^{\alpha-1} y(s)}{\Gamma(\alpha)} \mathrm{d} s .
$$

Substituting (2.6) into (2.5), we have

$$
\begin{aligned}
u(t)= & -\int_{0}^{t} \frac{(t-s)^{\alpha-1} y(s)}{\Gamma(\alpha)} \mathrm{d} s+\int_{0}^{1} \frac{t^{n-1}(1-s)^{\alpha-1} y(s)}{\Gamma(\alpha)} \mathrm{d} s \\
& \quad-\frac{n \lambda}{n-\lambda} \int_{0}^{1} \frac{t^{n-1}(1-s)^{\alpha} y(s)}{\Gamma(\alpha+1)} \mathrm{d} s+\frac{\lambda}{n-\lambda} \int_{0}^{1} \frac{t^{n-1}(1-s)^{\alpha-1} y(s)}{\Gamma(\alpha)} \mathrm{d} s \\
= & -\int_{0}^{t} \frac{(t-s)^{\alpha-1} y(s)}{\Gamma(\alpha)} \mathrm{d} s+\int_{0}^{1} \frac{n t^{n-1}(1-s)^{\alpha-1}(\alpha-\lambda+\lambda s) y(s)}{(n-\lambda) \Gamma(\alpha+1)} \mathrm{d} s \\
= & -\int_{0}^{t} \frac{n t^{n-1}(1-s)^{\alpha-1}(\alpha-\lambda+\lambda s)-(n-\lambda) \alpha(t-s)^{\alpha-1}}{(n-\lambda) \Gamma(\alpha+1)} y(s) \mathrm{d} s \\
& \quad+\int_{0}^{1} \frac{n t^{n-1}(1-s)^{\alpha-1}(\alpha-\lambda+\lambda s)}{(n-\lambda) \Gamma(\alpha+1)} y(s) \mathrm{d} s \\
= & \int_{0}^{1} G(t, s) y(s) \mathrm{d} s .
\end{aligned}
$$

The proof of Lemma 2.3 is completed.

Now, we present the following lemma, which will play major in the proofs of our main results.

Lemma 2.4. (Arzelà-Ascoli [17]) Let $D \subset X$ be a compact set with a sequence $\left\{x_{n}\right\} \subset D$ being uniformly bounded and equicontinuous, then the sequence has a uniformly convergent subsequence.

Lemma 2.5. ([18]) Let $X$ be a Banach space with $\Omega \subset X$ being open and bounded, $0 \in \Omega$. let $T: \bar{\Omega} \rightarrow X$ be a completely continuous operator such that

$$
\|T u\| \leq\|u\|, \forall u \in \partial \Omega .
$$

Then $T$ has a fixed point in $\bar{\Omega}$.

Lemma 2.6. (Krasnoselskii's fixed point theorem [18]) Let X be a Banach space with $\mathbb{M} \subset X$ being closed convex and nonempty. If the operator $A$ and $B$ satisfy the following conditions :

(a) $A x+B y \in \mathbb{M}$, wherever $x, y \in \mathbb{M}$;

(b) $A$ is compact and continuous ;

(c) B is a contraction mapping.

Then there exists $z \in \mathbb{M}$ such that $z=A z+B z$. 


\section{Main results}

In this section, we investigate the existence and uniqueness results of positive solutions for boundary value problem (1.1). First, we renew some notions. Let $E=C([0,1], \mathbb{R})$ denote the set of all continuous functions from $[0,1]$ into $\mathbb{R}$. Then $E$ is a Banach space endowed with the norm defined by

$$
\|u\|=\sup _{t \in[0,1]}|u(t)|
$$

Define the operator $F: E \rightarrow E$ as

$$
\begin{aligned}
(F u)(t)=\int_{0}^{1} G(t, s) f(s, u(s)) \mathrm{d} s & \\
=-\int_{0}^{t} & \frac{(t-s)^{\alpha-1} y(s)}{\Gamma(\alpha)} \mathrm{d} s+\int_{0}^{1} \frac{t^{n-1}(1-s)^{\alpha-1} y(s)}{\Gamma(\alpha)} \mathrm{d} s \\
& \quad-\frac{n \lambda}{n-\lambda} \int_{0}^{1} \frac{t^{n-1}(1-s)^{\alpha} y(s)}{\Gamma(\alpha+1)} \mathrm{d} s \\
& +\frac{\lambda}{n-\lambda} \int_{0}^{1} \frac{t^{n-1}(1-s)^{\alpha-1} y(s)}{\Gamma(\alpha)} \mathrm{d} s, \quad t \in[0,1] .
\end{aligned}
$$

It follows from Lemma 2.3 that the fixed points of the operator $F$ coincide with the solutions of fractional differential equation (1.1). Next, we will prove that the operator $F$ : $E \rightarrow E$ is completely continuous.

Lemma 3.1. The operator $F: E \rightarrow E$ defined by (3.1) is completely continuous.

Proof. By continuity of functions $f$ and $G(t, s)$, the operator $F$ is continuous. Let $\Omega \subset E$ be bounded. Then for any $t \in[0,1]$ and $u \in \Omega$, there exists a positive constant $K_{1}>0$ such that $|f(t, u)| \leq K_{1}$. From which, we can deduce that

$$
\begin{gathered}
|(F u)(t)| \leq \int_{0}^{t} \frac{(t-s)^{\alpha-1}}{\Gamma(\alpha)}|f(s, u(s))| \mathrm{d} s+\int_{0}^{1} \frac{t^{n-1}(1-s)^{\alpha-1}}{\Gamma(\alpha)}|f(s, u(s))| \mathrm{d} s \\
\quad+\frac{n \lambda}{n-\lambda} \int_{0}^{1} \frac{t^{n-1}(1-s)^{\alpha}}{\Gamma(\alpha+1)}|f(s, u(s))| \mathrm{d} s \\
\quad+\frac{\lambda}{n-\lambda} \int_{0}^{1} \frac{t^{n-1}(1-s)^{\alpha-1}}{\Gamma(\alpha)}|f(s, u(s))| \mathrm{d} s \\
\leq\left(\frac{t^{\alpha}}{\Gamma(\alpha+1)}+\frac{t^{n-1}}{\Gamma(\alpha+1)}+\frac{n \lambda}{n-\lambda} \cdot \frac{t^{n-1}}{\alpha \Gamma(\alpha+1)}+\frac{\lambda}{n-\lambda} \cdot \frac{t^{n-1}}{\Gamma(\alpha+1)}\right) K_{1}
\end{gathered}
$$




$$
\begin{aligned}
& \leq\left(\frac{1}{\Gamma(\alpha+1)}+\frac{1}{\Gamma(\alpha+1)}+\frac{n \lambda}{n-\lambda} \cdot \frac{1}{\alpha \Gamma(\alpha+1)}+\frac{\lambda}{n-\lambda} \cdot \frac{1}{\Gamma(\alpha+1)}\right) K_{1} \\
& =\frac{2 n \alpha+n \lambda-\alpha \lambda}{(n-\lambda) \alpha \Gamma(\alpha+1)} K_{1}=: K_{2}
\end{aligned}
$$

which implies that $\|(F u)(t)\| \leq K_{2}$. Analogously, for the derivative, we obtain that

$$
\begin{aligned}
\left|(F u)^{\prime}(t)\right|=\mid-\int_{0}^{t} \frac{(t-s)^{\alpha-2}}{\Gamma(\alpha-1)} f(s, u(s)) \mathrm{d} s & \quad+\int_{0}^{1} \frac{n(n-1) t^{n-2}(1-s)^{\alpha-1}(\alpha-\lambda+\lambda s)}{(n-\lambda) \Gamma(\alpha+1)} f(s, u(s)) \mathrm{d} s \mid \\
\leq & \int_{0}^{t} \frac{(t-s)^{\alpha-2}}{\Gamma(\alpha-1)}|f(s, u(s))| \mathrm{d} s \\
\quad & \quad \int_{0}^{1} \frac{n(n-1) t^{n-2}(1-s)^{\alpha-1}(\alpha-\lambda+\lambda s)}{(n-\lambda) \Gamma(\alpha+1)}|f(s, u(s))| \mathrm{d} s \\
\leq & \left(\frac{1}{\Gamma(\alpha-1)} \int_{0}^{t}(t-s)^{\alpha-2} \mathrm{~d} s+\frac{n(n-1) \alpha}{(n-\lambda) \Gamma(\alpha+1)} \int_{0}^{1}(1-s)^{\alpha-1} \mathrm{~d} s\right) K_{1} \\
\leq & \left(\frac{1}{\Gamma(\alpha)}+\frac{n(n-1)}{(n-\lambda) \Gamma(\alpha+1)}\right) K_{1}=: K_{3} .
\end{aligned}
$$

Therefore, for all $0 \leq t_{1}<t_{2} \leq 1$, one can deduce that

$$
\left|(F u)\left(t_{2}\right)-(F u)\left(t_{1}\right)\right|=\left|\int_{t_{1}}^{t_{2}}(F u)^{\prime}(s) \mathrm{d} s\right| \leq \int_{t_{1}}^{t_{2}}\left|(F u)^{\prime}(s)\right| \mathrm{d} s \leq K_{3}\left(t_{2}-t_{1}\right),
$$

which implies that the operator $F$ is equicontinuous on $[0,1]$. Thus, by Lemma 2.4 , the operator $F: E \rightarrow E$ defined by (3.1) is completely continuous.

Next we consider the following existence results.

Theorem 3.1. Assume $f \in C([0,1] \times \mathbb{R}, \mathbb{R})$ and $\lim _{u \rightarrow 0} f(t, u) / u=0$. Then problem (1.1) has at least one solution on $[0,1]$.

Proof. From $\lim _{u \rightarrow 0} f(t, u) / u=0$, there exist positive constants $\varepsilon>0$ and $\delta>0$ such that

$$
|f(t, u)| \leq \varepsilon|u|, \quad \text { for any } 0<|u|<\delta,
$$

where $\varepsilon$ satisfies

$$
\max _{t \in[0,1]}\left\{\frac{t^{\alpha}+t^{n-1}}{\Gamma(\alpha+1)}+\frac{(\alpha+n) \lambda t^{n-1}}{(n-\lambda) \alpha \Gamma(\alpha+1)}\right\} \varepsilon \leq 1 .
$$


Define $\Omega_{\delta}=\{u \in E:|u|<\delta\}$, taking $u_{0} \in \partial \Omega_{\delta}$, i.e., $u_{0} \in E$, and $\left|u_{0}\right|=\delta$. According to (3.1), (3.2) and (3.3), we can take

$$
\begin{aligned}
\left|\left(F u_{0}\right)(t)\right| & \leq \max _{t \in[0,1]}\left\{\frac{t^{\alpha}}{\Gamma(\alpha+1)}+\frac{t^{n-1}}{\Gamma(\alpha+1)}+\frac{n \lambda}{n-\lambda} \cdot \frac{t^{n-1}}{\alpha \Gamma(\alpha+1)}+\frac{\lambda}{n-\lambda} \cdot \frac{t^{n-1}}{\Gamma(\alpha+1)}\right\} \varepsilon\left|u_{0}\right| \\
& \leq \max _{t \in[0,1]}\left\{\frac{t^{\alpha}+t^{n-1}}{\Gamma(\alpha+1)}+\frac{(\alpha+n) \lambda t^{n-1}}{(n-\lambda) \alpha \Gamma(\alpha+1)}\right\} \varepsilon\left|u_{0}\right| \\
& \leq\left|u_{0}\right| .
\end{aligned}
$$

By virtue of Lemma 3.1, we know that $F$ is completely continuous. It follows from above that all conditions of Lemma 2.5 hold. Therefore, the boundary value problem (1.1) has at least one solution on $[0,1]$.

Theorem 3.2. Suppose $f \in C([0,1] \times \mathbb{R}, \mathbb{R})$. Moreover, there exist two positive real constants $L, \mu$ such that the following conditions hold:

$\left(H_{1}\right)\|f(t, u)-f(t, v)\| \leq L\|u-v\|, \quad \forall t \in[0,1], u, v \in E ;$

(H $2 L \frac{2 n \alpha+n \lambda-\alpha \lambda}{(n-\lambda) \alpha \Gamma(\alpha+1)} \leq \mu<1$.

Then the boundary problem (1.1) has a unique solution on $[0,1]$.

Proof. First, we show that $F: B_{r} \rightarrow B_{r}$. Define $L_{0}=\sup _{t \in[0,1]}\|f(t, 0)\|$ and select

$$
r \geq \frac{L_{0}}{1-\mu} \cdot \frac{2 n \alpha+n \lambda-\alpha \lambda}{(n-\lambda) \alpha \Gamma(\alpha+1)} .
$$

Define a closed ball in $E$ as $B_{r}=\{u \in E:\|u\| \leq r\}$. For $u \in B_{r}$, we have

$$
\begin{gathered}
\|(F u)(t)\| \leq \int_{0}^{t} \frac{(t-s)^{\alpha-1}}{\Gamma(\alpha)}\|f(s, u(s))\| \mathrm{d} s+\int_{0}^{1} \frac{t^{n-1}(1-s)^{\alpha-1}}{\Gamma(\alpha)}\|f(s, u(s))\| \mathrm{d} s \\
\quad+\frac{n \lambda}{n-\lambda} \int_{0}^{1} \frac{t^{n-1}(1-s)^{\alpha}}{\Gamma(\alpha+1)}\|f(s, u(s))\| \mathrm{d} s \\
\quad+\frac{\lambda}{n-\lambda} \int_{0}^{1} \frac{t^{n-1}(1-s)^{\alpha-1}}{\Gamma(\alpha)}\|f(s, u(s))\| \mathrm{d} s \\
\leq \int_{0}^{t} \frac{(t-s)^{\alpha-1}}{\Gamma(\alpha)}(\|f(s, u(s))-f(s, 0)\|+\|f(s, 0)\|) \mathrm{d} s \\
+\int_{0}^{1} \frac{t^{n-1}(1-s)^{\alpha-1}}{\Gamma(\alpha)}(\|f(s, u(s))-f(s, 0)\|+\|f(s, 0)\|) \mathrm{d} s
\end{gathered}
$$




$$
\begin{aligned}
& \quad+\frac{n \lambda}{n-\lambda} \int_{0}^{1} \frac{t^{n-1}(1-s)^{\alpha}}{\Gamma(\alpha+1)}(\|f(s, u(s))-f(s, 0)\|+\|f(s, 0)\|) \mathrm{d} s \\
& \quad+\frac{\lambda}{n-\lambda} \int_{0}^{1} \frac{t^{n-1}(1-s)^{\alpha-1}}{\Gamma(\alpha)}(\|f(s, u(s))-f(s, 0)\|+\|f(s, 0)\|) \mathrm{d} s \\
& \leq\left(L r+L_{0}\right)\left(\frac{t^{\alpha}}{\Gamma(\alpha+1)}+\frac{t^{n-1}}{\Gamma(\alpha+1)}+\frac{n \lambda}{n-\lambda} \cdot \frac{t^{n-1}}{\alpha \Gamma(\alpha+1)}+\frac{\lambda}{n-\lambda} \cdot \frac{t^{n-1}}{\Gamma(\alpha+1)}\right) \\
& \leq\left(L r+L_{0}\right)\left(\frac{1}{\Gamma(\alpha+1)}+\frac{1}{\Gamma(\alpha+1)}+\frac{n \lambda}{n-\lambda} \cdot \frac{1}{\alpha \Gamma(\alpha+1)}+\frac{\lambda}{n-\lambda} \cdot \frac{1}{\Gamma(\alpha+1)}\right) \\
& =\left(L r+L_{0}\right) \frac{2 n \alpha+n \lambda-\alpha \lambda}{(n-\lambda) \alpha \Gamma(\alpha+1)} \\
& \leq r, \quad
\end{aligned}
$$

which implies that $F: B_{r} \rightarrow B_{r}$. On the other hand, for all $u, v \in E, t \in[0,1]$, we have

$$
\begin{aligned}
\|(F u)(t)-(F v)(t)\| \leq \int_{0}^{t} \frac{(t-s)^{\alpha-1}}{\Gamma(\alpha)}(\|f(s, u(s))-f(s, v(s))\|) \mathrm{d} s \\
\quad+\int_{0}^{1} \frac{t^{n-1}(1-s)^{\alpha-1}}{\Gamma(\alpha)}(\|f(s, u(s))-f(s, v(s))\|) \mathrm{d} s \\
\quad+\frac{n \lambda}{n-\lambda} \int_{0}^{1} \frac{t^{n-1}(1-s)^{\alpha}}{\Gamma(\alpha+1)}(\|f(s, u(s))-f(s, v(s))\|) \mathrm{d} s \\
\quad+\frac{\lambda}{n-\lambda} \int_{0}^{1} \frac{t^{n-1}(1-s)^{\alpha-1}}{\Gamma(\alpha)}(\|f(s, u(s))-f(s, v(s))\|) \mathrm{d} s \\
\leq L\|u-v\|\left(\frac{t^{\alpha}}{\Gamma(\alpha+1)}+\frac{t^{n-1}}{\Gamma(\alpha+1)}+\frac{n \lambda}{n-\lambda} \cdot \frac{t^{n-1}}{\alpha \Gamma(\alpha+1)}+\frac{\lambda}{n-\lambda} \cdot \frac{t^{n-1}}{\Gamma(\alpha+1)}\right) \\
\leq L \\
\left.=L \frac{1}{\Gamma(\alpha+1)}+\frac{1}{\Gamma(\alpha+1)}+\frac{n \lambda}{n-\lambda} \cdot \frac{1}{\alpha \Gamma(\alpha+1)}+\frac{\lambda}{n-\lambda} \cdot \frac{1}{\Gamma(\alpha+1)}\right)\|u-v\| \\
=\frac{1}{(n-\lambda) \alpha \Gamma(\alpha+1)}\|u-v\|, \quad v \lambda \|
\end{aligned}
$$

which implies that $F$ is a contraction operator. Therefore, thank to the contraction mapping principle, the boundary value problem (1.1) has a unique solution on $[0,1]$. This completes the proof. 
Theorem 3.3. Let $f \in C([0,1] \times \mathbb{R}, \mathbb{R})$ satisfies the following conditions:

$\left(I_{1}\right)\|f(t, u)-f(t, v)\| \leq L\|u-v\|, \quad \forall t \in[0,1], u, v \in E ;$

$\left(I_{2}\right)\|f(t, u)\| \leq \varphi(t), \quad \forall t \in[0,1], u \in E$, and $\varphi \in L^{1}\left([0,1], \mathbb{R}^{+}\right)$,

where

$$
L \frac{n \alpha+n \lambda}{(n-\lambda) \alpha \Gamma(\alpha+1)}<1 .
$$

Then the boundary problem (1.1) has at least one solution on $[0,1]$.

Proof. Let

$$
r \geq\|\varphi\|_{L^{1}} \frac{2 n \alpha+n \lambda-\alpha \lambda}{(n-\lambda) \alpha \Gamma(\alpha+1)},
$$

and define a closed ball in $E$ as $B_{r}=\{u \in E:\|u\| \leq r\}$. Now, we define the operators $T_{1}$ and $T_{2}$ on $B_{r}$ as

$$
\begin{aligned}
&\left(T_{1} u\right)(t)=-\int_{0}^{t} \frac{(t-s)^{\alpha-1}}{\Gamma(\alpha)} f(s, u(s)) \mathrm{d} s \\
&\left(T_{2} u\right)(t)=\int_{0}^{1} \frac{t^{n-1}(1-s)^{\alpha-1}}{\Gamma(\alpha)} f(s, u(s)) \mathrm{d} s-\frac{n \lambda}{n-\lambda} \int_{0}^{1} \frac{t^{n-1}(1-s)^{\alpha}}{\Gamma(\alpha+1)} f(s, u(s)) \mathrm{d} s \\
& \quad+\frac{\lambda}{n-\lambda} \int_{0}^{1} \frac{t^{n-1}(1-s)^{\alpha-1}}{\Gamma(\alpha)} f(s, u(s)) \mathrm{d} s .
\end{aligned}
$$

For every $u, v \in B_{r}$, from (3.4) and (3.5), one can obtain that

$$
\left\|\left(T_{1} u\right)(t)+\left(T_{2} u\right)(t)\right\| \leq\|\varphi\|_{L^{1}} \frac{2 n \alpha+n \lambda-\alpha \lambda}{(n-\lambda) \alpha \Gamma(\alpha+1)} \leq r,
$$

which implies $T_{1}+T_{2} \in B_{r}$. From the proof of Theorem 3.2, we can take $T_{2}$ is a contraction operator. Since $f$ is continuous, $T_{1}$ is also continuous. In addition, for arbitrary $u \in B_{r}$, we find

$$
\left\|T_{1} u\right\| \leq \frac{t^{\alpha}}{\Gamma(\alpha+1)}\|\varphi\|_{L^{1}} \leq \frac{1}{\Gamma(\alpha+1)}\|\varphi\|_{L^{1}},
$$

by using $\left(I_{2}\right)$. Thus, $T_{1}$ is uniformly bounded on $B_{r}$. Next, we prove the compactness of the operator $T_{1}$. Let $\mathrm{S}=[0,1] \times B_{r}$, and define $f_{\max }=\sup _{(t, u) \in \mathrm{S}}\|f(t, u)\|$. For any $t_{1}, t_{2} \in[0,1]$, one can get

$$
\begin{aligned}
& \left\|\left(T_{1} u\right)\left(t_{2}\right)-\left(T_{1} u\right)\left(t_{1}\right)\right\|=\left\|\int_{0}^{t_{2}} \frac{\left(t_{2}-s\right)^{\alpha-1}}{\Gamma(\alpha)} f(s, u(s)) \mathrm{d} s-\int_{0}^{t_{1}} \frac{\left(t_{1}-s\right)^{\alpha-1}}{\Gamma(\alpha)} f(s, u(s)) \mathrm{d} s\right\| \\
= & \left\|\int_{0}^{t_{1}} \frac{f(s, u(s))}{\Gamma(\alpha)}\left(\left(t_{2}-s\right)^{\alpha-1}-\left(t_{1}-s\right)^{\alpha-1}\right) \mathrm{d} s-\int_{t_{1}}^{t_{2}} \frac{\left(t_{2}-s\right)^{\alpha-1}}{\Gamma(\alpha)} f(s, u(s)) \mathrm{d} s\right\|
\end{aligned}
$$




$$
\leq \frac{f_{\max }}{\Gamma(\alpha+1)}\left|2\left(t_{2}-t_{1}\right)^{\alpha}+t_{1}{ }^{\alpha}-t_{2}{ }^{\alpha}\right|,
$$

which is independent of $u$. We can obtain that $T_{1}$ is equicontinuous. Note that $f$ maps bounded subsets into relatively compact subsets, we can get that $T_{1}\left(E_{b s}\right)(t)$ is relatively compact in $E$ for all $t$, where $E_{b s} \subset E$ is bounded. Hence, $T_{1}(\cdot)$ is relatively compact on $B_{r}$. By the means of Arzelà-Ascoli theorem, $T_{1}$ is compact on $B_{r}$, Consequently, in view of Lemma 2.6, we conclude that the nonlinear boundary value problem (1.1) has at least one solution on $[0,1]$.

Theorem 3.4. Assume that $f \in C([0,1] \times \mathbb{R}, \mathbb{R})$. Moreover, there exist real constant $\eta$ satisfying

$$
0 \leq \eta<\frac{1}{\omega}, \quad \text { for } \omega=\frac{2 n \alpha+n \lambda-\alpha \lambda}{(n-\lambda) \alpha \Gamma(\alpha+1)} .
$$

Let constant $M>0$ such that $|f(t, u)| \leq \eta\|u\|+M$, for each $t \in[0,1], u \in \mathbb{R}$. Then the boundary problem (1.1) has at least one solution on $[0,1]$.

Proof. Let

$$
r=\frac{M \omega}{1-\eta \omega}+1
$$

and define a open ball in $E$ as

$$
B_{r}=\left\{u \in E: \max _{t \in[0,1]}|u| \leq r\right\} .
$$

Then, we only need to show that mapping $F u: \overline{B_{r}} \rightarrow E$ satisfies

$$
u \neq \rho F u, \quad \forall u \in \partial B_{r}, \rho \in[0,1] .
$$

Let $H(\rho, u)=\rho F u, u \in E, \rho \in[0,1]$. Then, it is easily show that $h_{\rho}(u)=u-H(\rho, u)=u-$ $\rho F u$ is completely continuous by Lemma 3.1. If (3.6) holds, according to the homotopy invariance of topological degree in Leray-Schauder degree theory, we deduce that

$$
\begin{aligned}
\operatorname{deg}\left(h_{\rho}, B_{r}, 0\right) & =\operatorname{deg}\left(I-\rho F u, B_{r}, 0\right)=\operatorname{deg}\left(h_{1}, B_{r}, 0\right)=\operatorname{deg}\left(h_{0}, B_{r}, 0\right) \\
& =\operatorname{deg}\left(I, B_{r}, 0\right)=1 \neq 0, \quad 0 \in B_{r},
\end{aligned}
$$

where $I$ denotes the unit operator. We can get that there exists $u \in B_{r}$ such as $h_{1}(u)=$ $u-\rho F u=0$ by using the nonzero property of the Leray-Schauder degree.

Next, we prove (3.6). Suppose that $u=\rho F u$ for some $\rho \in[0,1]$ and any $t \in[0,1]$. Then, we have

$$
|u(t)|=|\rho F u(t)| \leq \int_{0}^{t} \frac{(t-s)^{\alpha-1}}{\Gamma(\alpha)}|f(s, u(s))| \mathrm{d} s+\int_{0}^{1} \frac{t^{n-1}(1-s)^{\alpha-1}}{\Gamma(\alpha)}|f(s, u(s))| \mathrm{d} s
$$




$$
\begin{aligned}
& \quad+\frac{n \lambda}{n-\lambda} \int_{0}^{1} \frac{t^{n-1}(1-s)^{\alpha}}{\Gamma(\alpha+1)}|f(s, u(s))| \mathrm{d} s+\frac{\lambda}{n-\lambda} \int_{0}^{1} \frac{t^{n-1}(1-s)^{\alpha-1}}{\Gamma(\alpha)}|f(s, u(s))| \mathrm{d} s \\
& \leq(\eta\|u\|+M)\left(\int_{0}^{t} \frac{(t-s)^{\alpha-1}}{\Gamma(\alpha)} \mathrm{d} s+\int_{0}^{1} \frac{t^{n-1}(1-s)^{\alpha-1}}{\Gamma(\alpha)} \mathrm{d} s\right. \\
& \left.\quad+\frac{n \lambda}{n-\lambda} \int_{0}^{1} \frac{t^{n-1}(1-s)^{\alpha}}{\Gamma(\alpha+1)} \mathrm{d} s+\frac{\lambda}{n-\lambda} \int_{0}^{1} \frac{t^{n-1}(1-s)^{\alpha-1}}{\Gamma(\alpha)} \mathrm{d} s\right) \\
& \leq(\eta\|u\|+M)\left(\frac{t^{\alpha}}{\Gamma(\alpha+1)}+\frac{t^{n-1}}{\Gamma(\alpha+1)}+\frac{n \lambda}{n-\lambda} \cdot \frac{t^{n-1}}{\alpha \Gamma(\alpha+1)}+\frac{\lambda}{n-\lambda} \cdot \frac{t^{n-1}}{\Gamma(\alpha+1)}\right) \\
& \leq(\eta\|u\|+M)\left(\frac{1}{\Gamma(\alpha+1)}+\frac{1}{\Gamma(\alpha+1)}+\frac{n \lambda}{n-\lambda} \cdot \frac{1}{\alpha \Gamma(\alpha+1)}+\frac{\lambda}{n-\lambda} \cdot \frac{1}{\Gamma(\alpha+1)}\right) \\
& =(\eta\|u\|+M) \frac{2 n \alpha+n \lambda-\alpha \lambda}{(n-\lambda) \alpha \Gamma(\alpha+1)}=(\eta\|u\|+M) \omega .
\end{aligned}
$$

Straightforward computation gives

$$
\|u\| \leq \frac{M \omega}{1-\eta \omega}
$$

where $\|u\|=\sup _{t \in[0,1]}|u(t)|$. Note that

$$
r=\frac{M \omega}{1-\eta \omega}+1
$$

So (3.6) holds. This completes the proof.

\section{Examples}

In this section, we present several examples to illustrate our theoretical results.

Example 4.1. Consider the following problem of fractional differential equations with integral boundary conditions

$$
\left\{\begin{array}{l}
{ }^{c} D^{5 / 2} u(t)+t(\sin u-u)+\sqrt[3]{u^{2}+1}-1=0, \quad 0<t<1, \\
u(0)=u^{\prime \prime}(0)=0, u(1)=\frac{3}{2} \int_{0}^{1} u(s) \mathrm{d} s
\end{array}\right.
$$

from (4.1), we have $\alpha=5 / 2, \lambda=3 / 2, f(t, u)=t(\sin u-u)+\sqrt[3]{u^{2}+1}-1$. Also as $n=2$, $2<\alpha<3$ and $0<\lambda<2$. By straightforward calculation we get that $\lim _{u \rightarrow 0} f(t, u) / u=0$. Then, in virtue of Theorem 3.1, the boundary value problem (4.1) has at least one solution on $[0,1]$. 
Example 4.2. Consider the following problem of fractional differential equations with integral boundary conditions

$$
\left\{\begin{array}{l}
{ }^{c} D^{7 / 2} u(t)+\frac{\|u\|}{(t+2)(1+\|u\|)}=0, \quad 0<t<1, \\
u(0)=u^{\prime}(0)=u^{\prime \prime \prime}(0)=0, u(1)=2 \int_{0}^{1} u(s) \mathrm{d} s
\end{array}\right.
$$

where $\alpha=7 / 2, \lambda=2$,

$$
f(t, u)=\frac{\|u\|}{(t+2)(1+\|u\|)}
$$

as $n=3,3<\alpha<4,0<\lambda<3$. One can easily obtain that

$$
\|f(t, u)-f(t, v)\| \leq \frac{1}{2}\|u-v\| .
$$

When we choose $L=1 / 2$, a simple calculation gives

$$
2 L \frac{6 \alpha+3 \lambda-\alpha \lambda}{(3-\lambda) \alpha \Gamma(\alpha+1)} \approx 0.491266<1 .
$$

Obviously, the conditions $\left(H_{1}\right)$ and $\left(H_{2}\right)$ of Theorem 3.2 are satisfied, the boundary value problem (4.2) has a unique solution on $[0,1]$.

Example 4.3. Consider the following problem of fractional differential equations with integral boundary conditions

$$
\begin{cases}{ }^{c} D^{19 / 4} u(t)+\frac{\|u\|}{(t+3)(1+\|u\|)}=0, & 0<t<1, \\ u(0)=u^{\prime}(0)=u^{\prime \prime}(0)=u^{(4)}(0)=0, & u(1)=\frac{10}{3} \int_{0}^{1} u(s) \mathrm{d} s,\end{cases}
$$

in this case, $\alpha=19 / 4, \lambda=10 / 3$,

$$
f(t, u)=\frac{\|u\|}{(t+3)(1+\|u\|)}
$$

as $n=4,4<\alpha<5,0<\lambda<4$. We can obtain that

$$
\|f(t, u)-f(t, v)\| \leq \frac{1}{3}\|u-v\| .
$$

When we choose $L=\frac{1}{3}$, by standard calculation, we have

$$
L \frac{8 \alpha+4 \lambda-\alpha \lambda}{(4-\lambda) \alpha \Gamma(\alpha+1)} \approx 0.047431<1
$$


Moreover

$$
\|f(t, u)\| \leq \varphi(t)=\frac{1}{t+3} \in L^{1}\left([0,1], \mathbb{R}^{+}\right) .
$$

Clearly, all the assumptions of Theorem 3.3 are satisfied, the boundary problem (4.3) has at least one solution on $[0,1]$.

Example 4.4. Consider the following problem of fractional differential equations with integral boundary conditions

$$
\begin{cases}{ }^{c} D^{23 / 4} u(t)+\frac{\|u\|}{(t+4)(1+\|u\|)}=0, & 0<t<1, \\ u(0)=u^{\prime}(0)=u^{\prime \prime}(0)=u^{(4)}(0)=0, & u(1)=4 \int_{0}^{1} u(s) \mathrm{d} s,\end{cases}
$$

according to (4.4), we have $\alpha=15 / 4, \lambda=2$, as $n=3,3<\alpha<4,0<\lambda<3$,

$$
f(t, u)=\frac{\|u\|}{(t+4)(1+\|u\|)} .
$$

We can obtain that

$$
\omega=\frac{2 n \alpha+n \lambda-\alpha \lambda}{(n-\lambda) \alpha \Gamma(\alpha+1)} \approx 0.337630
$$

by straightforward calculation. Then, it is easy to see that there exists infinitely many positive constant $M$, such that

$$
|f(t, u)| \leq \frac{\|u\|}{4}+M \leq \frac{\|u\|}{\omega}+M, \quad \forall t \in[0,1], u \in \mathbb{R} .
$$

Thus, it follows from Theorem 3.4 that the boundary value problem (4.4) has at least one solution on $[0,1]$.

\section{Acknowledgement}

Our work was supported by National Natural Science Foundation of China (11301454), the TianYuan Special Funds of the National Natural Science Foundation of China (115261 -77), Natural Science Foundation of Jiangsu Province (BK20151160), the Natural Science Foundation for Colleges and Universities in Jiangsu Province (14KJB110025) and the Project of Xuzhou University of Technology (XKY2017113).

\section{References}

[1] Shah K., Khan R.A., Existence and uniqueness of positive solutions to a coupled system of nonlinear fractional order differential equations with anti periodic boundary conditions. Differ. Equ. Appl., 7 (2) (2015), 245-262. 
[2] Graef R, Kong L., Positive solutions for a class of higher order boundary value problems with fractional q-derivatives. Applied Mathematics and Computation., 218 (19) (2012), 96829689.

[3] Zhang H., Zheng Z., Jiang W., Existence of solutions for nonlinear fractional order functional differential equations. Acta Mathematica Scientia (Series A)., 31 (2) (2011), 289-297.

[4] Zhang X., Feng M., Positive solutions for a second-order differential equation with integral boundary conditions and deviating arguments. Boundary Value Problems., 2015 (222) (2015), $1-21$.

[5] Zhang H., Wu D., Variation of constant formulae for time invariant and time varying fractional delay differential systems. Journal of Mathematical Research with Applications., 34 (5) (2014), 549-560.

[6] Sun Y., Zhao M., Positive solutions for a class of fractional differential equations with integral boundary conditions. Applied Mathematics Letters., 34 (1) (2014), 17-21.

[7] Liang J., Liu L., Wu Y., Second-order nonlinear singular Sturm-Liouville problems with integral boundary conditions. Applied Mathematics and Computation., 215 (4) (2009), 1573-1582.

[8] Xu Y., Fractional Boundary Value Problems with Integral and Anti-periodic Boundary Conditions. Bulletin of the Malaysian Mathematical Society., 39 (2) (2016), 571-587.

[9] Wang L., Zhang X., Existence of positive solutions for a class of higher-order nonlinear fractional differential equations with integral boundary conditions and a parameter. Journal of Applied Mathematics and Computing., 44 (1-2) (2014), 293-316.

[10] Bai Z., L H., Positive solutions for boundary value problem of nonlinear fractional order differential equations. Journal of Mathematical Analysis and Applications., 311 (2) (2005), 495505.

[11] Xu X., Jiang D., Yuan C., Multiple positive solutions for the boundary value problem of a nonlinear fractional differential equation. Nonlinear Analysis., 71 (10) (2009), 4676-4688.

[12] Cabada A, Wang G T. Positive solutions of nonlinear fractional differential equations with integral boundary value conditions. Journal of Mathematical Analysis and Applications., 389 (1) (2012), 403-411.

[13] Zhang Z., Liu J.B., Cao J., et al, Stability results for the linear degenerate fractional differential system. Advances in Difference Equations., 2016 (216) (2016), 1-17.

[14] Zhang H., Wu D., Variation of constant formulae for time invariant and time varying fractional delay differential systems. Journal of Mathematical Research with Applications., 34 (5) (2014), 549-560.

[15] Kilbas A., Srivastava H., Trujillo J., Theory and Applications of Fractional Differential Equations. Elsevier, Amsterdam, 2006.

[16] Podlubny I., Fractional Differential Equations. Academic Press, San Diego, 1999.

[17] Deimling K., Nonlinear Functional Analysis. Springer, Berlin, 1985.

[18] Smart D.R., Fixed Point Theorems. Cambridge University Press, Cambridge, 1980. 